\title{
Diversidad y distribución vertical de líquenes corticícolas en la pluvisilva montana de la Gran Piedra, Cuba
}

\author{
Dania Rosabal ${ }^{1}$, Ana Rosa Burgaz ${ }^{2}$ Orlando J. Reyes ${ }^{1}$
}

Resumen: Rosabal, D.; Burgaz, A. R. \& Reyes, O. J. 2012. Diversidad y distribución vertical de líquenes corticícolas en la pluvisilva montana de la Gran Piedra, Cuba. Bot. Complut. 36: 19-30.

\begin{abstract}
Los líquenes epífitos que crecen en los árboles de la pluvisilva montana de la Gran Piedra (Cuba) muestran claramente una distribución vertical sobre los troncos. Se han establecido nueve cuadrados de $625 \mathrm{~m}^{2}$ de cinco forófitos diferentes. Cada árbol se dividió en cuatro zonas desde la base hasta las ramas más altas. Se han encontrado 115 especies de las que hay 50 nuevas para Cuba. Las especies de biotipo crustáceo con reproducción sexual son las más frecuentes a lo largo de todo el árbol. La familia Thelotremataceae domina en la base de los troncos (zona I) mientras que Graphidaceae y Parmeliaceae son más abundantes en las ramas más altas. Las zonas II, III y IV poseen la mayor diversidad de especies.
\end{abstract}

Palabras clave: Neotrópico, líquenes del Caribe, epífitos.

Abstract: Rosabal, D.; Burgaz, A. R. \& Reyes, O. J. 2012. Diversity and vertical distribution of corticolous lichens in the montane rainforest of the Gran Piedra, Cube. Bot. Complut. 36: 19-30.

The epiphytic lichen flora in the montane rainforest of the Gran Piedra (Cube) shows a clear vertical distribution. Nine cuadrats of $625 \mathrm{~m}^{2}$ were established and thirty chosen trees of five different species were surveyed. Each tree was divided in four zones from the ground to highest external branches. One hundred and fifteen corticolous lichen species were recorded, including 50 newly recorded for Cuba. Crustose species with sexual reproduction strategies were the commonest along the tree. Thelotremataceae family was dominant at the tree bases (zone I) while Graphidaceae and Parmeliaceae were more abundant in the highest branches. The zones II, III and IV harbour the highest lichen diversity.

Key words: Neotropic, Caribbean lichens, epiphytes.

\section{INTRODUCCIÓN}

Estudios ecológicos en el Neotrópico han demostrado que los líquenes no están distribuidos de manera aleatoria sobre el árbol, sino que responden a una pronunciada estratificación vertical (Cornelissen \& ter Steege 1989, Sipman \& Harris 1989, Benzing 1990, Montfoort \& Ek 1990, Komposch \& Hafellner 2000, Gradstein et al. 2003). Esta zonación puede estar influida por las condiciones de humedad, luz y temperatura, las cuales varían en un gradiente desde la base del árbol hasta el dosel (Wolseley \& Aguirre-Hudson 1997). La mayoría de las especies de líquenes se encuentran por encima de los 2 m de altura (Komposch \& Hafellner 2000), lo cual realza la importancia del estudio de la distribución vertical de los líquenes para propósitos de conservación. Johans- son (1974) propone un esquema de zonación vertical con seis zonas de altura, desarrollado originalmente para grandes árboles de un bosque lluvioso del oeste africano y está basado principalmente en la estructura del forófito. Este esquema es el más comúnmente usado en estudios de distribución vertical de líquenes en bosques tropicales (Cornelissen \& ter Steege 1989, Montfoort \& Ek 1990, Komposch \& Hafellner 2000).

En Cuba, los trabajos sobre ecología de líquenes son escasos (Rosabal et al. 2010) y estudios sobre su distribución vertical son desconocidos. El objetivo de la investigación es determinar la variación de la composición, riqueza y frecuencia de líquenes en un gradiente vertical de altura desde la base del árbol hasta las ramas superiores en cinco especies de forófitos en la pluvisilva montana de la Gran Piedra.

\footnotetext{
${ }^{1}$ Departamento de Biología, Universidad de Oriente. Patricio Lumumba s/n. CP. 90500, Cuba.drl@cnt.ou.edu.cu

${ }^{2}$ Departamento de Biología Vegetal I, Facultad de Ciencias Biológicas, Universidad Complutense de Madrid, E-28040 Madrid, Spain. arburgaz@bio.ucm.es

Recibido: 21 mayo 2012. Aceptado: 6 junio 2012.
} 


\section{MATERIALES Y MÉTODOS}

El Paisaje Natural Protegido Gran Piedra pertenece a la Reserva de la Biosfera Baconao en este se localiza la pluvisilva montana de la Gran Piedra, en las coordenadas $75^{\circ} 37^{\prime} 47^{\prime \prime}$ y $20^{\circ} 0^{\prime} 42^{\prime \prime}$ (Fig. 1). La temperatura media anual es de $18,4^{\circ} \mathrm{C}$ y la media anual de las precipitaciones es de $1664 \mathrm{~mm}$ (Montenegro 1991). La humedad relativa promedia es de $89,6 \%$; condicionado fundamentalmente por la ocurrencia de las nieblas, el uso de un cortarramas (tubo plástico de $4 \mathrm{~m}$ de longitud con una tijera podadora en el extremo).

Las especies fueron identificadas mediante claves especializadas (Hale 1976, 1987; Wirth \& Hale 1978; Moberg 1990; Brako 1991; Galloway 1994; Lumbsch et al. 1999; Brodo et al. 2001; Rivas Plata et al. 2006; Aptroot et al. 2008, 2009). Los cortes de talo y estructuras reproductoras se realizaron bajo el microscopio estereoscopio Novel NTB-2B (4x) y la observación y medición de las esporas se realizó bajo el microscopio

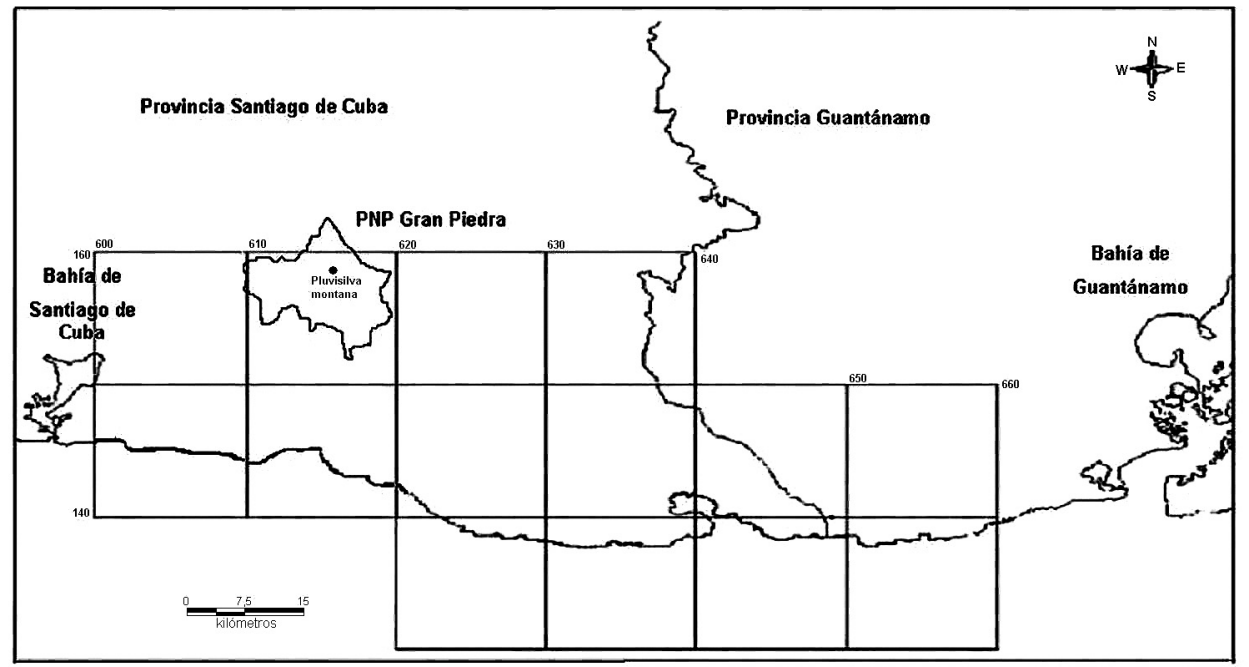

Fig. 1- Ubicación geográfica del Paisaje Natural Protegido Gran Piedra. El punto señala la ubicación de la pluvisilva montana de la Gran Piedra.

las cuales se presentan como promedio 238 días al año (Montenegro 1991).

La altura del estrato arbóreo es de 12-18 m, clasificado como un bosque bajo a medio (Reyes et al. 2005). Las especies de árboles Myrcia fenzliana, Coccoloba wrightii, Clusia tetrastigma, Dendropanax arboreus y Brunellia comocladifolia son forófitos con una elevada abundancia de individuos en este bosque.

El trabajo se llevó a cabo desde marzo de 2010 hasta marzo de 2011. Se establecieron nueve parcelas cuadradas de 625 $\mathrm{m}^{2}$ y en cada una se seleccionó uno de los árboles presentes de las cinco especies de forófitos, según su disponibilidad. Cada árbol se dividió en cuatro zonas de altura (Fig. 2). La zona I se extendió desde el suelo hasta los $2 \mathrm{~m}$ de altura sobre el tronco. La zona II (dosel interno) desde 2-4 m, seleccionando las ramas que presentaron un diámetro mayor de $15 \mathrm{~cm}$. La zona III (dosel medio) desde los $4 \mathrm{~m}$ hasta la mitad de la distancia al extremo terminal de las ramas, se seleccionaron ramas de diámetro entre $5-15 \mathrm{~cm}$. La zona IV desde el límite de la zona III hasta el extremo de las ramas externas; en este nivel se escogieron, aleatoriamente, diez ramas con diámetros entre $1-3 \mathrm{~cm}$. En las zonas II y III el muestreo se realizó trepando sobre el árbol con ayuda de una cuerda. La recolecta en la zona IV se facilitó por

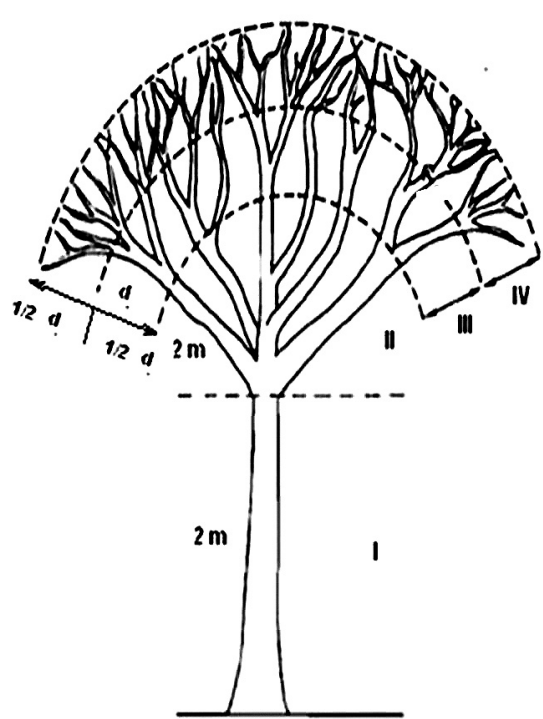

Fig. 2-Esquema de zonación vertical propuesto para las cinco especies de forófitos en la pluvisilva montana. d: distancia desde los cuatro metros hasta el extremo terminal de las ramas. 
óptico Novel XSZ-N207 (400x). Para la actualización de la taxonomía de las especies de líquenes se ha seguido el criterio de Kirk (2008). Los materiales colectados fueron depositados en el Herbario Charles Ramsden del Departamento de Biología de la Universidad de Oriente, sección docente del Herbario Dr. Jorge Sierra Calzado (BSC) del Centro Oriental de Ecosistemas y Biodiversidad.

Para determinar la variación de la composición de especies de líquenes entre las cuatro zonas de altura se utilizó el método de ordenación Non-metric Multidimensional Scaling (NMS), contenido en el programa PC-Ord 5.0 para Windows (McCune $\&$ Mefford 1999). Para comprobar diferencias en cuanto a riqueza y frecuencia de especies entre las zonas se empleó la prueba Kruskal-Wallis con comparaciones múltiples a priori, según el criterio de Siegel \& Castellán (1995) y para el análisis se usó el paquete estadístico STATISTICA 6.0.

\section{RESULTADOS}

La composición de líquenes corticícolas sobre los 30 árboles de las cinco especies de forófitos, muestreados desde la base del tronco hasta las ramas externas de la copa, se relaciona en la Tabla 1. Se encontraron 115 especies de líquenes, las cuales pertenecen a 43 géneros de 24 familias. Las familias con mayor número de especies son Graphidaceae (20), Parmeliaceae (16) y Thelotremataceae (13). Se registraron 50 nuevas especies para Cuba (Tabla 1). Los líquenes crustáceos predominaron en todas las zonas de altura. El número de especies con biotipo foliáceo aumentó hacia las zonas II, III y IV. Las especies con biotipos escuamuloso y filamentoso predominan en las zonas I y II. Los líquenes fruticulosos se encuentran restringidos a las zonas III y IV (Fig. 3).

En cuanto a las estrategias reproductivas, las especies con reproducción por esporas predominaron en todas las zonas. Las especies con reproducción vegetativa (isidios o soredios) aumentaron su número hacia las zonas II, III y IV (Fig. 4). No se encontraron especímenes con los dos tipos de reproducción en el mismo talo.

La composición de líquenes mostró variación en relación con las cuatro zonas de altura (Fig. 5). Se encontró un patrón de segregación de especies hacia las zonas I y IV, evidenciando la mayor disimilitud entre estas dos zonas.

Los valores de riqueza y frecuencia de líquenes entre las zonas se muestran en la Tabla 2, no se encontraron diferencias significativas en cuanto a estas variables entre zonas. De las 115 especies registradas, $83(72,2 \%)$ se hallaron exclusivamente por encima de los $2 \mathrm{~m}$ de altura.

La zona I fue la que presentó menor riqueza y frecuencia de líquenes (Tabla 2). En esta zona, la especie con ma- yor frecuencia fue Porina distans (11), seguida de Herpothallon rubrocinctum (10). El 46,9\% de las especies de líquenes tuvieron el mínimo valor de frecuencia. Los géneros con mayor número de especies fueron Coenogonium (4), Ocellularia (3), Phyllopsora (3), Leptogium (3), Porina (3) y Graphis (3). La familia con la mayor riqueza específica fue Thelotremataceae representada por cinco especies de líquenes distribuidas en tres géneros.

Los mayores valores de riqueza y frecuencia de líquenes se registraron en la zona II (Tabla 2). Las especies Sticta weigelii (7) y Ocellularia dolichotata (7) fueron las de mayor frecuencia. El 56,9\% de las especies registradas en esta zona presentaron el menor valor de frecuencia y los géneros Ocellularia y Coenogonium aumentaron su riqueza a seis especies cada uno. Otros géneros que contribuyen a la diversidad de líquenes en esta área del árbol son Pertusaria, Graphis, Leptogium, Porina y Parmotrema. Thelotremataceae, con nueve especies, fue la familia con la mayor riqueza específica en esta zona.

En la zona III, los líquenes con mayor frecuencia fueron Hypotrachyna subphysodalica, Leptogium cyanescens y Parmotrema tinctorum (Tabla 2). El 60\% de las especies de líquenes presentaron el valor mínimo de frecuencia. Graphis es el género con mayor número de especies (7), le sigue Pertusaria con seis especies. En esta zona, predomina la familia Graphidaceae con 11 especies distribuidas en tres géneros, le continúa en importancia Parmeliaceae con nueve especies pertenecientes a tres géneros.

La especie Brigantiaea leucoxantha presentó el mayor valor de frecuencia en la zona IV, le continúan Hypotrachyna subphysodalica, Pertusaria pustulata y Graphis rustica (Tabla 2). El 59,7\% de las especies presentaron el menor valor de frecuencia (1). El género Graphis es el que mayor número de especies presenta. Las familias Graphidaceae (16) y Parmeliaceae (11) aumentan la diversidad de especies en esta zona.

De manera general, la frecuencia de los líquenes dominantes disminuye mientras que la frecuencia total de especies aumenta desde la zona I hacia la zona IV; así como, el porcentaje de especies con el valor mínimo de frecuencia aumenta hacia la zona IV. Los géneros OceIlularia, Coenogonium y Phyllopsora presentaron mayor riqueza y frecuencia hacia las zonas I y II del árbol mientras que Graphis, Parmotrema y Pertusaria aumentan estos parámetros hacia las zonas III y IV. La familia Thelotremataceae incrementa su riqueza y frecuencia de líquenes hacia la base del árbol, en tanto Graphidaceae y Parmeliaceae lo hacen hacia los estratos superiores. 
Tabla 1

Lista de especies de líquenes. C: crustáceo; E: escuamuloso; F: foliáceo; Fi: filamentoso; Fr: fruticuloso. * Nuevo registro de especie para Cuba

\begin{tabular}{|c|c|c|c|c|}
\hline Familias & Especies de líquenes & Abreviaturas & Biotipo & Fotobionte \\
\hline Arthoniaceae & $\begin{array}{l}\text { *Arthonia orbygniae Bat. \& J. L. Bezerra } \\
\text { Arthothelium spectabile A. Massal. } \\
\text { *Arthonia lecythidicola (Bat. \& H. Maia) Lücking } \\
\text { \& Sérus. } \\
\text { Cryptothecia striata Thor } \\
\text { Herpothallon rubrocinctum (Ehrenb) Aptroot, } \\
\text { Lücking \& G. Thor }\end{array}$ & $\begin{array}{l}\text { Arth-orb } \\
\text { Athth-sp } \\
\text { Arth-lec } \\
\text { Cryp-str } \\
\text { Herp-rub }\end{array}$ & $\begin{array}{l}\mathrm{C} \\
\mathrm{C} \\
\mathrm{C}\end{array}$ & $\begin{array}{l}\text { Trentepohlia } \\
\text { Trentepohlia } \\
\text { Trentepohlia } \\
\text { Trentepohlia } \\
\text { Trentepohlia }\end{array}$ \\
\hline Arthopyreniaceae & $\begin{array}{l}\text { *Mycomicrothelia punctata Aptroot } \\
\text { *Mycomicrothelia subfallens (Müll. Arg.) } \\
\text { D. Hawksw. }\end{array}$ & $\begin{array}{l}\text { Mycom-pu } \\
\text { Mycom-su }\end{array}$ & $\mathrm{C}$ & $\begin{array}{l}\text { Trentepohlia } \\
\text { Trentepohlia }\end{array}$ \\
\hline Atheliaceae & Dictyonema glabratum (Spreng.) D. Hawksw. & Dict-gla & $\mathrm{F}$ & Scytonema \\
\hline Bacidiaceae & $\begin{array}{l}\text { Phyllopsora furfuracea Zahlbr. } \\
\text { Phyllopsora buettneri (Müll. Arg.) Zahlbr. } \\
\text { Phyllopsora corallina (Eschw.) Müll. Arg. } \\
\text { Phyllopsora parvifolia (Pers.) Müll. Arg. }\end{array}$ & $\begin{array}{l}\text { Phyl-fur } \\
\text { Phyl-bue } \\
\text { Phyl-cor } \\
\text { Phyl-par }\end{array}$ & $\begin{array}{l}\mathrm{E} \\
\mathrm{E} \\
\mathrm{E} \\
\mathrm{E}\end{array}$ & $\begin{array}{l}\text { Pseudochlorella } \\
\text { Pseudochlorella } \\
\text { Pseudochlorella } \\
\text { Pseudochlorella }\end{array}$ \\
\hline Brigantiaceae & $\begin{array}{l}\text { Brigantiaea leucoxantha (Spreng.) R. Sant. } \\
\& \text { Hafellner }\end{array}$ & Brig-leu & $\mathrm{C}$ & Clorobionte \\
\hline Byssolomataceae & Byssoloma leucoblepharum (Nyl.) Vain. & Byss-leu & $\mathrm{C}$ & Clorobionte \\
\hline Chrysothricaceae & Chrysothrix candelaris (L.) J. R. Laundon & Chry-can & $\mathrm{C}$ & Clorobionte \\
\hline Coccocarpiaceae & $\begin{array}{l}\text { Coccocarpia palmicola (Spreng.) Arv. \& } \\
\text { D. J. Galloway }\end{array}$ & Coc-palm & $\mathrm{F}$ & Scytonema \\
\hline Coenogoniaceae & $\begin{array}{l}\text { Coenogonium disjunctum Nyl. } \\
\text { Coenogonium interplexum Nyl. } \\
\text { Coenogonium interpositum Nyl. } \\
\text { Coenogonium leprieurii (Mont.) Nyl. } \\
\text { Coenogonium linkii Ehrenb. } \\
\text { Coenogonium pusillum (Mont.) Lücking, Aptroot } \\
\text { \& Sipman }\end{array}$ & $\begin{array}{l}\text { Coe-disj } \\
\text { Coe-ipl } \\
\text { Coe-ipo } \\
\text { Coe-lepr } \\
\text { Coe-link } \\
\text { Coe-pus }\end{array}$ & $\begin{array}{l}\mathrm{Fi} \\
\mathrm{Fi} \\
\mathrm{Fi} \\
\mathrm{Fi} \\
\mathrm{Fi}\end{array}$ & $\begin{array}{l}\text { Trentepohlia } \\
\text { Trentepohlia } \\
\text { Trentepohlia } \\
\text { Trentepohlia } \\
\text { Trentepohlia } \\
\text { Trentepohlia }\end{array}$ \\
\hline Collemataceae & $\begin{array}{l}\text { Leptogium marginellum (Sw.) Gray } \\
\text { Leptogium austroamericanum (Malme) C.W. Dodge } \\
\text { Leptogium azureum (Sw. ex Ach.) Mont. } \\
\text { Leptogium cyanescens (Pers.) Körb. }\end{array}$ & $\begin{array}{l}\text { Lept-mar } \\
\text { Lept-aus } \\
\text { Lept-azu } \\
\text { Lept-cya }\end{array}$ & $\begin{array}{l}F \\
F \\
F \\
F\end{array}$ & $\begin{array}{l}\text { Nostoc } \\
\text { Nostoc } \\
\text { Nostoc } \\
\text { Nostoc }\end{array}$ \\
\hline Crocyniaceae & Crocynia pyxinoides Nyl. & Cro-pyxi & $\mathrm{C}$ & Trentepohlia \\
\hline Graphidaceae & $\begin{array}{l}\text { *Diorygma hieroglyphicum (Pers.) Staiger \& Kalb } \\
\text { Diorygma poitaei (Fée) Kalb, Staiger \& Elix. } \\
\text { *Fissurina dumastoides (Fink) Staiger } \\
\text { *Fissurina incrustans Fée }\end{array}$ & $\begin{array}{l}\text { Dior-hie } \\
\text { Dior-poi } \\
\text { Fiss-dum } \\
\text { Fiss-inc }\end{array}$ & $\begin{array}{l}\mathrm{C} \\
\mathrm{C} \\
\mathrm{C} \\
\mathrm{C}\end{array}$ & $\begin{array}{l}\text { Trentepohlia } \\
\text { Trentepohlia } \\
\text { Trentepohlia } \\
\text { Trentepohlia }\end{array}$ \\
\hline
\end{tabular}




\begin{tabular}{|c|c|c|c|c|}
\hline Familias & Especies de líquenes & Abreviaturas & Biotipo & Fotobionte \\
\hline & *Graphis acharii Fée & Grap-ach & $\mathrm{C}$ & Trentepohlia \\
\hline & Graphis adpressa Vain. & Grap-adp & $\mathrm{C}$ & Trentepohlia \\
\hline & Graphis anfractuosa (Eschw.) Eschw. & Grap-anf & $\mathrm{C}$ & Trentepohlia \\
\hline & *Graphis antillarum Vain. & Grap-ant & $\mathrm{C}$ & Trentepohlia \\
\hline & *Graphis chlorocarpa Fée & Grap-chl & $\mathrm{C}$ & Trentepohlia \\
\hline & Graphis chrysocarpa (Raddi) Spreng. & Grap-chr & $\mathrm{C}$ & Trentepohlia \\
\hline & *Graphis descuamescens Fée & Grap-des & $\mathrm{C}$ & Trentepohlia \\
\hline & *Graphis flexibilis Kremp. & Grap-fle & $\mathrm{C}$ & Trentepohlia \\
\hline & *Graphis humilis Vain. & Grap-hum & $\mathrm{C}$ & Trentepohlia \\
\hline & *Graphis illinata Eschw. & Grap-ill & $\mathrm{C}$ & Trentepohlia \\
\hline & *Graphis imshaugii M. Wirth \& Hale & Grap-ims & $\mathrm{C}$ & Trentepohlia \\
\hline & *Graphis macella Kremp. & Grap-mac & $\mathrm{C}$ & Trentepohlia \\
\hline & Graphis rhizocola (Fée) Lücking \& Chaves & Grap-rhi & $\mathrm{C}$ & Trentepohlia \\
\hline & Graphis rustica Kremp. & Grap-rus & $\mathrm{C}$ & Trentepohlia \\
\hline & Platythecium grammitis (Fée) Staiger & Plat-gra & $\mathrm{C}$ & Trentepohlia \\
\hline & *Sarcographa difformis (Vain.) Zahlbr. & Sarc-dif & $\mathrm{C}$ & Trentepohlia \\
\hline \multirow[t]{2}{*}{ Monoblasticeae } & *Anisomeridium americanum (A. Massal.) R. C. Harris & Anis-ame & $\mathrm{C}$ & Trentepohlia \\
\hline & *Anisomeridium ambiguum(Zahlbr.) R. C. Harris & Anis-amb & $\mathrm{C}$ & Trentepohlia \\
\hline \multirow[t]{16}{*}{ Parmeliaceae } & Canoparmelia caroliniana (Nyl.) Elix \& Hale & Cano-car & $\mathrm{F}$ & Trebouxia \\
\hline & Hypotrachyna costaricensis (Nyl.) Hale & Hyp-cost & $\mathrm{F}$ & Trebouxia \\
\hline & Hypotrachyna lineariloba (Kurok.) Hale & Hyp-line & $\mathrm{F}$ & Trebouxia \\
\hline & Hypotrachyna livida (Taylor) Hale & Hyp-liv & $\mathrm{F}$ & Trebouxia \\
\hline & Hypotrachyna lopezii Hale & Hyp-lop & $\mathrm{F}$ & Trebouxia \\
\hline & Hypotrachyna subphysodalica (Hale) Hale & Hyp-subp & $\mathrm{F}$ & Trebouxia \\
\hline & Parmotrema crinitum (Ach.) M. Choisy & Parm-cri & $\mathrm{F}$ & Trebouxia \\
\hline & Parmotrema cristiferum (Taylor) Hale & Parm-crs & $\mathrm{F}$ & Trebouxia \\
\hline & *Parmotrema hypotropum (Nyl.) Hale & Parm-hyp & $\mathrm{F}$ & Trebouxia \\
\hline & *Parmotrema michauxianum (Zahlbr.) Hale & Parm-mic & $\mathrm{F}$ & Trebouxia \\
\hline & *Parmotrema xanthinum (Müll. Arg.) Hale & Parm-xan & $\mathrm{F}$ & Trebouxia \\
\hline & Parmotrema tinctorum (Despr. ex Nyl.) Hale & Parm-tin & $\mathrm{F}$ & Trebouxia \\
\hline & * Rimelia cetrata (Ach.) Hale \& A. Fletcher & Rim-cetr & $\mathrm{F}$ & Trebouxia \\
\hline & *Rimelia reticulata (Taylor) Hale \& A. Fletcher & Rim-ret & $\mathrm{F}$ & Trebouxia \\
\hline & Usnea hirta (L.) Weber ex F.H. Wigg. & Usn-hirt & $\mathrm{Fr}$ & Trebouxia \\
\hline & Usnea rubicunda Stirt. & Usn-rub & $\mathrm{Fr}$ & Trebouxia \\
\hline \multirow[t]{8}{*}{ Pertusariaceae } & *Pertusaria albescens (Huds.) M. Choisy \& Werner & Pert-alb & $\mathrm{C}$ & Trebouxia \\
\hline & *Pertusaria amara (Ach.) Nyl. & Pert-ama & $\mathrm{C}$ & Trebouxia \\
\hline & Pertusaria azulensis B. de Lesd. & Pert-azu & $\mathrm{C}$ & Trebouxia \\
\hline & *Pertusaria pustulata (Ach.) Duby & Pert-pu & $\mathrm{C}$ & Trebouxia \\
\hline & *Pertusaria rubefacta Erichsen & Pert-rub & $\mathrm{C}$ & Trebouxia \\
\hline & *Pertusaria tetrathalamia (Fée) Nyl. & Pert-tet & $\mathrm{C}$ & Trebouxia \\
\hline & *Pertusaria texana Müll. Arg. & Pert-tex & $\mathrm{C}$ & Trebouxia \\
\hline & *Pertusaria xanthodes Müll. Arg. & Pert-xan & $\mathrm{C}$ & Trebouxia \\
\hline \multirow[t]{2}{*}{ Physciaceae } & *Physcia atrostriata Moberg & Phys-atr & $\mathrm{F}$ & Trebouxia \\
\hline & *Physcia lopezii Moberg & Phys-lop & $\mathrm{F}$ & Trebouxia \\
\hline
\end{tabular}




\begin{tabular}{|c|c|c|c|c|}
\hline Familias & Especies de líquenes & Abreviaturas & Biotipo & Fotobionte \\
\hline & Heterodermia leucomela (L.) Poelt & Het-leuc & $\mathrm{F}$ & Trebouxia \\
\hline & Heterodermia obscurata (Nyl.) Trevis. & Het-obsc & $\mathrm{F}$ & Trebouxia \\
\hline \multirow[t]{6}{*}{ Porinaceae } & Porina americana Fée & Por-ame & $\mathrm{C}$ & Trentepohlia \\
\hline & *Porina conspersa Malme & Por-cons & $\mathrm{C}$ & Trentepohlia \\
\hline & *Porina rudiuscula (Nyl.) Müll. Arg. & Por-rudi & $\mathrm{C}$ & Trentepohlia \\
\hline & Porina distans Vězda \& Vivant & Por-dist & $\mathrm{C}$ & Trentepohlia \\
\hline & Porina mastoidea Fée & Por-mast & $\mathrm{C}$ & Trentepohlia \\
\hline & Porina nucula Ach. & Por-nuc & $\mathrm{C}$ & Trentepohlia \\
\hline \multirow[t]{7}{*}{ Pyrenulaceae } & *Anthracothecium macrosporum (Hepp) Müll. Arg. & Anth-mac & $\mathrm{C}$ & Trentepohlia \\
\hline & * Lithothelium illotum (Nyl.) Aptroot & Lith-ill & $\mathrm{C}$ & Trentepohlia \\
\hline & *Pyrenula dermatodes (Borrer) Schaer. & Pyr-derm & $\mathrm{C}$ & Trentepohlia \\
\hline & *Pyrenula mastophoroides (Nyl.) Zahlbr. & Pyr-mph & $\mathrm{C}$ & Trentepohlia \\
\hline & Pyrenula macrocarpa A. Massal. & Pyr-macr & $\mathrm{C}$ & Trentepohlia \\
\hline & Pyrenula santesis (Nyl.) Müll. Arg. & Pyr-sant & $\mathrm{C}$ & Trentepohlia \\
\hline & Pyrenula subcongruens Müll. Arg. & Pyr-subc & $\mathrm{C}$ & Trentepohlia \\
\hline Ramalinaceae & *Ramalina stenospora Müll. Arg. & Ram-sten & $\mathrm{Fr}$ & Trebouxia \\
\hline Stereocaulaceae & *Lepraria lobificans Nyl. & Lepr-lob & $\mathrm{C}$ & Clorobionte \\
\hline \multirow[t]{2}{*}{ Stictaceae } & Sticta beauvoisii Delise & Stic-bea & $\mathrm{F}$ & Nostoc \\
\hline & Sticta weigelii Isert & Stic-wei & $\mathrm{F}$ & Nostoc \\
\hline Teloschistaceae & Teloschistes exilis (Michx.) Vain. & Telo-exi & $\mathrm{Fr}$ & Trebouxia \\
\hline \multirow[t]{13}{*}{ Thelotremataceae } & *Myriotrema microporellum (Nyl.) Hale & Myr-micr & $\mathrm{C}$ & Trentepohlia \\
\hline & Myriotrema costaricense (Müll. Arg.) Hale & Myr-cost & $\mathrm{C}$ & Trentepohlia \\
\hline & Ocellularia antillensis Hale & Oce-anti & $\mathrm{C}$ & Trentepohlia \\
\hline & *Ocellularia chiriquiensis (Hale) Hale & Ocel-chi & $\mathrm{C}$ & Trentepohlia \\
\hline & Ocellularia crocea (Kremp.) Overeem \& D. Overeem & Ocel-cro & $\mathrm{C}$ & Trentepohlia \\
\hline & Ocellularia dolichotata (Nyl.) Zahlbr. & Ocel-dol & $\mathrm{C}$ & Trentepohlia \\
\hline & Ocellularia perforata (Leight.) Müll. Arg. & Ocel-per & $\mathrm{C}$ & Trentepohlia \\
\hline & Ocellularia praestans (Müll. Arg.) Hale & Ocel-pra & $\mathrm{C}$ & Trentepohlia \\
\hline & Ocellularia xanthostroma (Nyl.) Zahlbr. & Ocel-xan & $\mathrm{C}$ & Trentepohlia \\
\hline & *Stegobolus subemersus (Müll. Arg.) Frisch & Steg-sub & $\mathrm{C}$ & Trentepohlia \\
\hline & Thelotrema adjectum Nyl. & The-adj & $\mathrm{C}$ & Trentepohlia \\
\hline & Thelotrema lepadinum (Ach.) Ach. & The-lepa & $\mathrm{C}$ & Trentepohlia \\
\hline & *Thelotrema occlusum Nyl. & The-occl & $\mathrm{C}$ & Trentepohlia \\
\hline \multirow[t]{7}{*}{ Trypetheliaceae } & *Architrypethelium hyalinum Aptroot & Arch-hya & $\mathrm{C}$ & Trentepohlia \\
\hline & *Megalotremis biocellata Aptroot & Mega-bio & $\mathrm{C}$ & Trentepohlia \\
\hline & *Megalotremis lateralis Aptroot & Mega-lat & $\mathrm{C}$ & Trentepohlia \\
\hline & *Polymeridium quinqueseptatum (Nyl.) R. C. Harris & Poly-qui & $\mathrm{C}$ & Trentepohlia \\
\hline & *Polymeridium subcinereum (Nyl.) R. C. Harris & Poly-sub & $\mathrm{C}$ & Trentepohlia \\
\hline & Pseudopyrenula subnudata Müll. Arg. & Pseu-sub & $\mathrm{C}$ & Trentepohlia \\
\hline & Trypethelium subcatervarium Malme & Try-subc & $\mathrm{C}$ & Trentepohlia \\
\hline
\end{tabular}




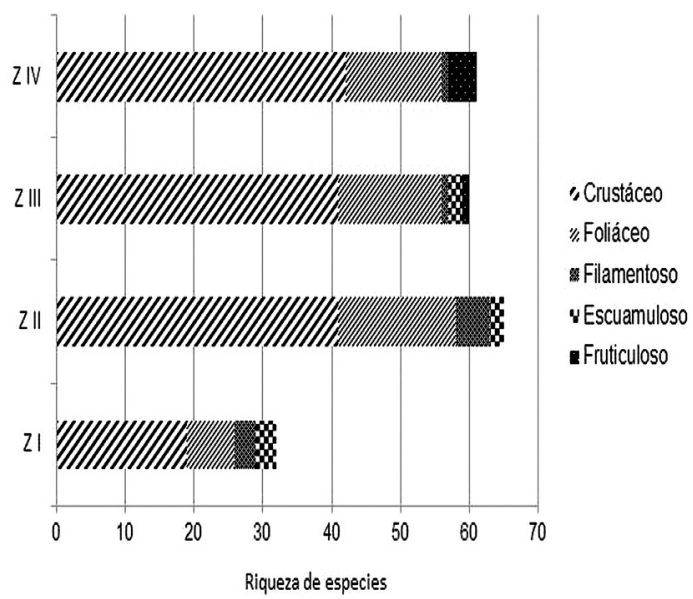

Fig. 3-Riqueza de especies por biotipos en las cuatro zonas de altura.

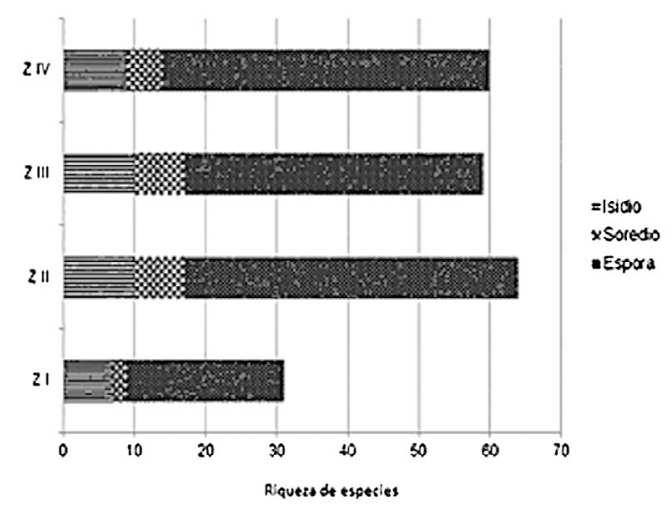

Fig. 4- Riqueza de especies por estrategias reproductivas en las cuatro zonas de altura.

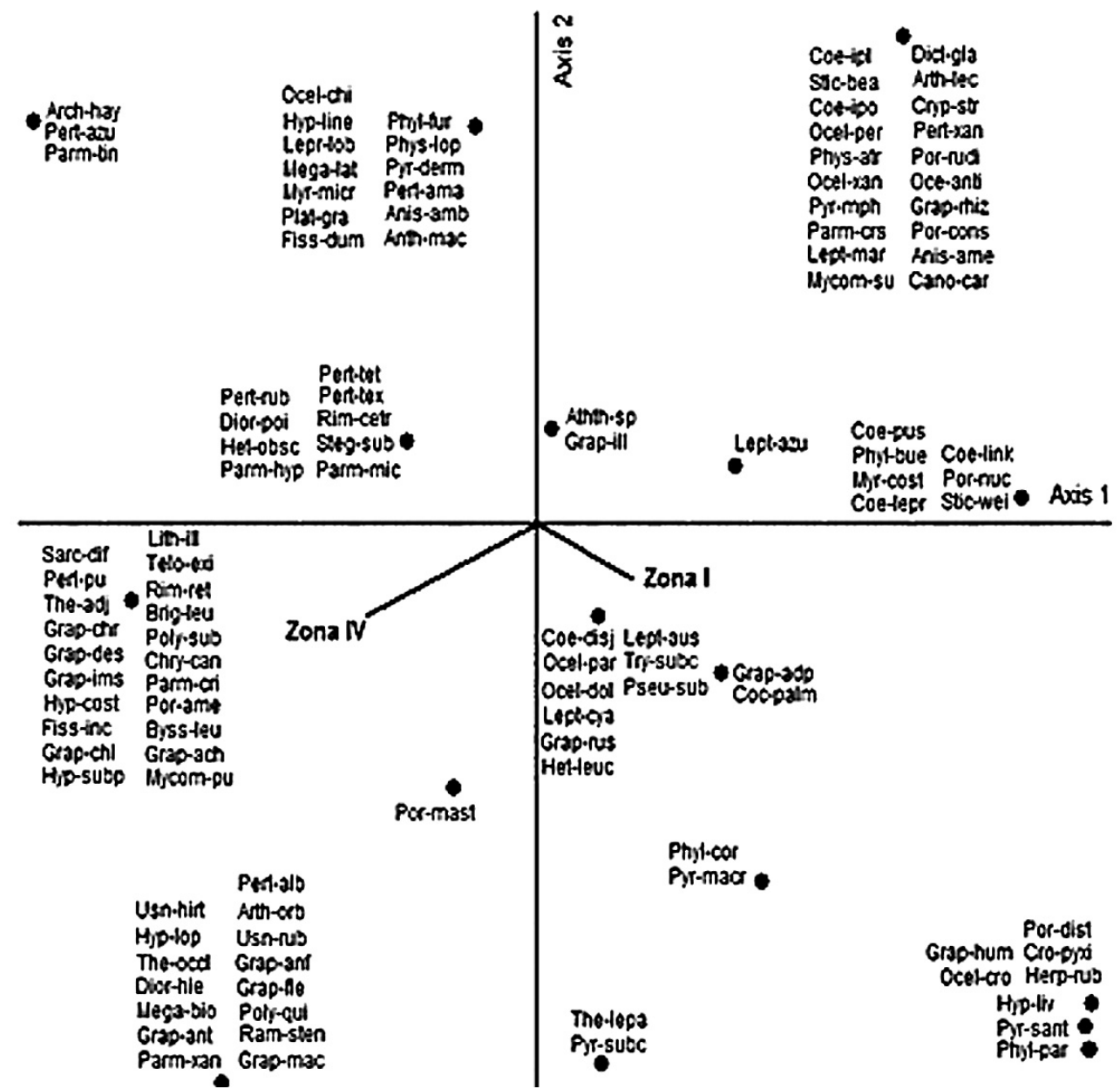

Fig. 5-Composición de especies de líquenes en las cuatro zonas de altura. La dirección y longitud de las líneas indican el sentido y la fortaleza de la correlación con las especies de líquenes (puntos). 
Tabla 2

Riqueza y frecuencia de especies de líquenes desde el suelo hasta las ramas externas de la copa en las cinco especies de forófitos. Z: zona

\begin{tabular}{|c|c|c|c|c|}
\hline Especies de líquenes & Z I & Z II & Z III & Z IV \\
\hline Anisomeridium americanum & 0 & 1 & 0 & 0 \\
\hline Anisomeridum ambiguun & 0 & 1 & 2 & 0 \\
\hline Anthracothecium macrosporum & 0 & 1 & 1 & 0 \\
\hline Architrypethelium hyalinum & 0 & 0 & 1 & 0 \\
\hline Arthonia lecytidicola & 0 & 1 & 0 & 0 \\
\hline Arthonia orbygniae & 0 & 0 & 0 & 1 \\
\hline Arthothelium spectabile & 0 & 2 & 0 & 3 \\
\hline Brigantiaea leucoxantha & 0 & 0 & 2 & 5 \\
\hline Byssoloma leucoblepharum & 0 & 0 & 1 & 2 \\
\hline Canoparmelia caroliniana & 0 & 2 & 0 & 0 \\
\hline Chrysotrix candelaris & 0 & 0 & 3 & 2 \\
\hline Coccocarpia palmicola & 1 & 6 & 0 & 1 \\
\hline Coenogonium disjunctum & 2 & 2 & 2 & 2 \\
\hline Coenogonium interplexum & 0 & 1 & 0 & 0 \\
\hline Coenogonium interpositum & 0 & 1 & 0 & 0 \\
\hline Coenogonium leprieurii & 2 & 2 & 0 & 0 \\
\hline Coenogonium linkii & 1 & 2 & 0 & 0 \\
\hline Coenogonium pusillum & 2 & 2 & 0 & 0 \\
\hline Crocynia pyxinoides & 4 & 0 & 0 & 0 \\
\hline Cryptotecia striata & 0 & 1 & 0 & 0 \\
\hline Dictyonema glabratum & 0 & 1 & 0 & 0 \\
\hline Diorygma hieroglyphicum & 0 & 0 & 0 & 1 \\
\hline Diorygma poitaei & 0 & 1 & 1 & 3 \\
\hline Fissurina dumastoides & 0 & 1 & 1 & 0 \\
\hline Fissurina incrustans & 0 & 0 & 1 & 1 \\
\hline Graphis acharii & 0 & 0 & 2 & 3 \\
\hline Graphis adpresa & 2 & 1 & 0 & 1 \\
\hline Graphis anfractuosa & 0 & 0 & 0 & 1 \\
\hline Graphis antillarum & 0 & 0 & 0 & 1 \\
\hline Graphis chlorocarpa & 0 & 0 & 1 & 1 \\
\hline Graphis chrysocarpa & 0 & 0 & 1 & 1 \\
\hline Graphis descuamescens & 0 & 0 & 1 & 1 \\
\hline Graphis flexibilis & 0 & 0 & 0 & 1 \\
\hline Graphis humilis & 1 & 0 & 0 & 0 \\
\hline Graphis illinata & 0 & 1 & 0 & 1 \\
\hline Graphis imshaugii & 0 & 0 & 1 & 1 \\
\hline Graphis macella & 0 & 0 & 0 & 1 \\
\hline Graphis rhizocola & 0 & 2 & 0 & 0 \\
\hline Graphis rustica & 2 & 1 & 3 & 4 \\
\hline Herpothallon rubrocinctum & 10 & 5 & 0 & 0 \\
\hline Heterodermia leucomela & 4 & 4 & 1 & 2 \\
\hline Heterodermia obscurata & 0 & 1 & 1 & 1 \\
\hline Hypotrachyna costaricensis & 0 & 0 & 3 & 2 \\
\hline Hypotrachyna lineariloba & 0 & 1 & 1 & 0 \\
\hline
\end{tabular}

\begin{tabular}{lcccc}
\hline Especies de líquenes & Z I & Z II & Z III & Z IV \\
\hline Hypotrachyna livida & 1 & 0 & 0 & 0 \\
Hypotrachyna lopezii & 0 & 0 & 0 & 1 \\
Hypotrachyna subphysodalica & 0 & 0 & 5 & 4 \\
Lepraria lobificans & 0 & 3 & 1 & 0 \\
Leptogium austroamericanum & 5 & 3 & 1 & 1 \\
Leptogium azureum & 1 & 2 & 1 & 0 \\
Leptogium cyanescens & 1 & 6 & 4 & 1 \\
Leptogium marginellum & 0 & 1 & 0 & 0 \\
Lithothelium illotum & 0 & 0 & 1 & 1 \\
Megalotremis biocellata & 0 & 0 & 0 & 2 \\
Megalotremis lateralis & 0 & 2 & 2 & 0 \\
Mycomicrothelia punctata & 0 & 0 & 1 & 1 \\
Mycomicrothelia subfallens & 0 & 2 & 0 & 0 \\
Myriotrema costarisensis & 2 & 1 & 0 & 0 \\
Myriotrema microporellum & 0 & 3 & 1 & 0 \\
Ocellularia antillensis & 0 & 1 & 0 & 0 \\
Ocellularia chiriquiensis & 0 & 3 & 1 & 0 \\
Ocellularia crocea & 1 & 0 & 0 & 0 \\
Ocellularia dolichotata & 1 & 7 & 3 & 2 \\
Ocellularia perforata & 0 & 2 & 0 & 0 \\
Ocellularia praestans & 2 & 2 & 3 & 1 \\
Ocellularia xanthostroma & 0 & 1 & 0 & 0 \\
Parmotrema crinitum & 0 & 0 & 1 & 2 \\
Parmotrema cristiferum & 0 & 1 & 0 & 0 \\
Parmotrema hypotropum & 0 & 2 & 1 & 1 \\
Parmotrema michauxianum & 0 & 1 & 2 & 1 \\
Parmotrema ticntorum & 0 & 0 & 4 & 0 \\
Parmotrema xanthinum & 0 & 0 & 0 & 1 \\
Pertusaria albescens & 0 & 0 & 0 & 1 \\
Pertusaria amara & 0 & 1 & 1 & 0 \\
Pertusaria azulensis & 0 & 0 & 2 & 0 \\
Pertusaria pustulata & 0 & 0 & 3 & 4 \\
Pertusaria rubefacta & 0 & 1 & 2 & 1 \\
Pertusaria tetrathalamia & 0 & 1 & 1 & 2 \\
Pertusaria texana & 0 & 1 & 2 & 1 \\
Pertusaria xanthodes & 0 & 1 & 0 & 0 \\
Phyllopsora buettneri & 6 & 1 & 0 & 0 \\
Phyllopsora corallina & 2 & 0 & 1 & 0 \\
Phyllopsora furfuracea & 0 & 1 & 1 & 0 \\
Phyllopsora parvifolia & 5 & 0 & 0 & 0 \\
Physcia atrostriata & 0 & 1 & 0 & 0 \\
Physcia lopezii & 0 & 1 & 1 & 0 \\
Platythecium grammitis & 0 & 1 & 1 & 0 \\
Polymeridium quinqueseptatum & 0 & 0 & 0 & 2 \\
\hline & & & &
\end{tabular}




\begin{tabular}{lcccc}
\hline Especies de líquenes & Z I & Z II & Z III & Z IV \\
\hline Polymeridium subcinereum & 0 & 0 & 1 & 3 \\
Porina americana & 0 & 0 & 2 & 1 \\
Porina conspersa & 0 & 1 & 0 & 0 \\
Porina distans & 11 & 3 & 1 & 0 \\
Porina mastoidea & 4 & 0 & 2 & 1 \\
Porina nucula & 1 & 3 & 0 & 0 \\
Porina rudiuscula & 0 & 2 & 0 & 0 \\
Pseudopyrenula subnudata & 3 & 1 & 3 & 1 \\
Pyrenula dermatodes & 0 & 1 & 1 & 0 \\
Pyrenula macrocarpa & 1 & 0 & 2 & 0 \\
Pyrenula mastophoroides & 0 & 1 & 0 & 0 \\
Pyrenula santesis & 1 & 0 & 0 & 0 \\
Pyrenula subcongruens & 1 & 0 & 0 & 2 \\
Ramalina stenospora & 0 & 0 & 0 & 3 \\
Rimelia cetrata & 0 & 1 & 1 & 1 \\
\hline
\end{tabular}

\begin{tabular}{lrrrr}
\hline Especies de líquenes & Z I & Z II & Z III & Z IV \\
\hline Rimelia reticulata & 0 & 0 & 1 & 1 \\
Sarcographa difformis & 0 & 0 & 1 & 2 \\
Stegobolus subemersus & 0 & 3 & 2 & 1 \\
Sticta beauvoisii & 0 & 3 & 0 & 0 \\
Sticta weigelii & 1 & 7 & 0 & 0 \\
Teloschistes exilis & 0 & 0 & 1 & 1 \\
Thelotrema adjectum & 0 & 0 & 1 & 1 \\
Thelotrema lepadinum & 1 & 0 & 0 & 1 \\
Thelotrema occlusum & 0 & 0 & 0 & 2 \\
Trypethelium subcatervarium & 1 & 1 & 2 & 1 \\
Usnea hirta & 0 & 0 & 0 & 2 \\
Usnea rubicunda & 0 & 0 & 0 & 2 \\
\hline Riqueza & $\mathbf{3 2}$ & $\mathbf{6 5}$ & $\mathbf{6 0}$ & $\mathbf{6 2}$ \\
\hline Frecuencia & $\mathbf{8 3}$ & $\mathbf{1 2 4}$ & $\mathbf{9 8}$ & $\mathbf{1 0 0}$ \\
\hline
\end{tabular}

\section{DISCUSIÓN}

Según Frisch et al. (2006), la familia Thelotremataceae constituye un componente importante de la biota liquénica epifítica de los bosques montanos, tendiendo a decrecer en la vegetación xeromorfa y en bosques a bajas altitudes. Coppins \& Wolseley (2002) destacan a esta familia como una de las dominantes en los estratos bajos de los bosques tropicales asiáticos. La presencia de especies de esta familia ha sido asociada a ecosistemas poco perturbados y es rara en la vegetación secundaria 0 antropogénica (Frisch et al. 2006; Rivas Plata et al. 2007).

Según Lücking \& Rivas-Plata (2008), Graphidaceae es una de las familias de líquenes con mayor número de especies y muestra una gran amplitud ecológica, ocupando diversos tipos de hábitats (Wirth \& Hale 1978). El elevado número de especies de esta familia puede estar relacionado con el tipo de fotobionte que participa en la simbiosis (Trentepohlia), muy frecuente en las formaciones vegetales tropicales (Sipman \& Harris, 1989). La reproducción en este grupo se realiza por esporas de origen sexual. Este tipo de diáspora puede ser propagada fácilmente por el viento, el agua y pequeños animales (moluscos, insectos), lo cual le permite llegar a una mayor cantidad de micrositios. El éxito de la colonización de estos hábitats se halla potenciado por la alta disponibilidad de Trentepohlia, el fotobionte específico de esta familia. Además, todas las especies desarrollan talos crustáceos, lo cual constituye una ventaja adaptativa tanto en ambientes húmedos como los bosques lluviosos montanos (Lakatos

et al. 2006). Rosabal \& Aragón (2010), encontraron que Graphidaceae fue la de mayor riqueza de especies en el matorral xeromorfo costero de la Reserva Ecológica Siboney-Juticí, corroborando la alta plasticidad ecológica de la familia.

Las especies con biotipo crustáceo predominaron en todas las zonas de alturas. En el tronco, donde la humedad es elevada, esta forma de crecimiento limita la retención de agua y escurre más rápidamente el exceso, lo cual permite alcanzar niveles adecuados de hidratación del talo. Este parámetro influye sobre el intercambio de gases y la fotosíntesis neta, donde por encima del $80 \%$ de saturación del talo, la fotosíntesis neta comienza a disminuir (Nash 1996, Purvis 2000). En las ramas externas de la copa del árbol, las especies de líquenes crustáceos también son abundantes. Este biotipo, fuertemente adherido al sustrato reduce el área de exposición y limita la pérdida de agua por evaporación (Jahns 1988). Cornelissen \& ter Steege (1989), Montfoort \& Ek (1990) y Komposch \& Hafellner (2000) obtuvieron patrones similares en cuanto a las formas de crecimiento en las zonas de altura sobre el árbol.

Los líquenes fruticulosos fueron exclusivos de las ramas del dosel medio y externo. Büdel \& Scheidegger (1996) plantean que las especies con este biotipo son encontradas preferencialmente en ecosistemas con climas muy húmedos. La morfología de los talos, altamente ramificados y separados del sustrato, les provee una mayor superficie en proporción a su volumen. Dicha característica resulta en un aumento de la velocidad de hidratación y desecación del talo. 
Según Barkman (1958), las especies del género Usnea frecuentemente están restringidas a la región de la copa de los árboles en bosques densos. Este autor plantea que la evaporación en la copa del árbol es, generalmente, más fuerte que en las zonas bajas. Esto se produce por el efecto del viento, el cual aumenta su velocidad con la altura sobre el suelo. Nash (1996) destaca la capacidad de la mayoría de los líquenes de recuperar completamente los niveles metabólicos después de períodos prolongados de desecación. Esto ha sido probado en el liquen fruticuloso Ramalina maciformis (Lange 1969). No obstante, en la pluvisilva montana la humedad relativa es elevada, por lo que los períodos de desecación de los talos son más cortos, comparado con los que podrían experimentarse en otros ambientes. Probablemente, estos cortos períodos de desecación del talo favorecen el establecimiento de estas especies fotófilas en las ramas externas con un bajo costo metabólico.

Las especies con reproducción por esporas prevalecieron en todas las zonas del árbol. No obstante, las especies productoras de isidios y soredios se distribuyeron en todo el gradiente vertical. Las esporas son diásporas dispersadas fácilmente por el viento, el agua y pequeños animales. La gran cantidad de agentes dispersores aumenta las probabilidades de colonización de microhábitats disponibles por estas especies. Barkman (1958), planteó que los propágulos vegetativos, fundamentalmente los isidios hidrofílicos, son transportados por el agua de lluvia. Esto promueve una extensión de la población a lo largo del tronco y dificulta la dispersión a larga distancia. Komposch \& Hafellner (2002), hallaron que la reproducción por esporas es predominante hacia el dosel y la reproducción vegetativa es una estrategia más frecuente en las especies del sotobosque. El patrón observado en la pluvisilva montana difiere del encontrado por esos autores.

La composición de especies mostró una estratificación vertical bien definida desde la base del árbol hasta las ramas externas del dosel. En la zona I (hasta los $2 \mathrm{~m}$ ) se encontraron líquenes de los géneros Porina, Leptogium y Coenogonium. Según Sipman y Harris (1989), las especies de estos géneros son tolerantes a las condiciones de baja iluminación y por tanto dominantes en las zonas bajas de los árboles en los bosques tropicales. La familia Thelotremataceae junto a géneros como Phyllopsora y Crocynia, así como otros líquenes crustáceos estériles, por ejemplo Herpothallon rubrocinctum, pueden llegar a alcanzar gran diversidad en esta zona (Sipman \& Harris 1989). Además de la tolerancia a los bajos niveles de iluminación, estas especies se encuentran favorecidas por las adaptaciones que presentan a la elevada humedad en los estratos más bajos del árbol. Los resultados difieren de los obtenidos por Komposch \& Hafellner (2000), quienes encontraron un patrón de dominancia de especies diferente para este estrato.

La riqueza y frecuencia total de líquenes disminuyó hacia la zona I. Esta zona tiene una mayor abundancia de líquenes crustáceos y foliáceos que desarrollan grandes talos formando manchas que cubren la superficie del tronco y disminuyendo el espacio disponible para la colonización de nuevas especies. Además, en esta zona se encuentra una gran abundancia de briófitos que compiten exitosamente por el hábitat. La combinación de estos factores presupone una disminución de la riqueza y frecuencia de especies de líquenes hacia el estrato más bajo del árbol así como el establecimiento de una composición de especies características de esta zona por sus capacidades de adaptación a las condiciones del microhábitat. Por otro lado, el tronco es un microhábitat continuo, por lo que las especies favorecidas por la continuidad de las condiciones existentes en este estrato excluirán a otras especies, diminuyendo la diversidad de líquenes sobre el árbol. Según Wolf (1995), la dominancia de especies está asociada a los últimos estadios sucesionales. Los resultados obtenidos coinciden con los encontrados por Hinds (1970), Eversman et al. (1987), Komposch \& Hafellner (2000) y Fanning et al. (2007).

La mayoría de las especies se hallaron por encima de los $2 \mathrm{~m}$ de altura sobre el tronco, en las zonas II, III y IV. La zona II tuvo la mayor riqueza de especies, la cual presenta las ramas de mayor diámetro en comparación con las del dosel medio y externo. En esta zona hay una mayor superficie y ha permanecido por más tiempo expuesta a la llegada de las diásporas. Por otro lado, esta es una zona donde los parámetros microambientales no son extremos, lo cual puede favorecer la colonización de numerosas especies. Este resultado podría estar determinado por una mayor diversidad de microhábitats, si se tiene en cuenta que estas son zonas intermedias donde factores como iluminación y humedad no son extremos. Según Barkman (1958), el microclima es menos extremo desde la periferia de la copa hacia la base de la copa, donde decrece la velocidad del viento y la intensidad luminosa.

Además, el grosor y ramificación de las ramas aumenta el área expuesta a la llegada de propágulos. MacArthur \& Wilson (1967), postularon la teoría de la biogeografía de islas, donde predicen que la diversidad de especies aumenta con el aumento del área de superficie. Wolf (1995), plantea que las ramas de mayor diámetro han permanecido expuesta a la llegada de las diásporas por más tiempo. Este autor encontró un aumento de la riqueza de especies 
hacia las ramas de mayor diámetro en la copa de los árboles, lo cual coincide con los resultados obtenidos.

La zona IV estuvo dominada por especies de la familia Graphidaceae. Komposch \& Hafellner (2000) obtuvieron resultados similares, sugiriendo una amplia plasticidad ecológica dentro de la familia. Las zonas II, III y IV tuvieron una elevada riqueza y frecuencia de especies. Según Hubbell (2001), la diversidad de especies se encuentra favorecida por la fragmentación del nicho y la dispersión altamente estocástica de las diásporas, como mecanismos que evitan la exclusión de especies equivalentes. Las ramas del dosel interno, medio y externo son más delgadas y altamente ramificadas, si las comparamos con el tronco, lo que posibilita que el espacio y las condiciones del microhábitat no sean continuas, permitiendo la llegada aleatoria de las diásporas y el establecimiento de diferentes especies en diferentes fragmentos del mismo nicho.

\section{CONCLUSIONES}

Los líquenes en la pluvisilva montana de la Gran Piedra muestran una pronunciada estratificación vertical. El talo crustáceo en combinación con la reproducción sexual es la estrategia más frecuente desde la base del tronco hasta la copa del árbol. Thelotremataceae predominó hacia la base, disminuyendo su diversidad en las ramas externas. Graphidaceae y Parmeliaceae, por el contrario, aumentan su diversidad hacia la copa del árbol. La mayor riqueza de líquenes se encontró por encima de los dos metros de altura. Las zonas I y IV presentaron la mayor disimilitud en la composición de especies.

\section{AGRADECIMIENTOS}

Queremos agradecer a Robert Lücking las correcciones en la nomenclatura de algunas especies.

\section{BIBLIOGRAFÍA}

Aptroot, A.; Thor, G.; Lücking, R.; Elix, J. A. \& Chávez, J. L. 2009. The lichen genus Herpothallon reinstated. Biblioth. Lichenol. 99: 19-66.

Aptroot, A.; LÜCKIng, R.; SiPMAN, H.; UmaÑA, L. \& ChÁveZ, J. 2008. Pyrenocarpous lichens with bitunicate asci. A first assessment of the lichen biodiversity inventory in Costa Rica: Biblioth. Lichenol. 97: 1-162.

Barkman, J. J. 1958. Phytosociology and ecology of cryptogamic epiphytes. Van Gorcum, Assen.

Benzing, D. H. 1990. Vascular epiphytes: General biology and related biota. Cambridge. Univ. Press, New York.

Brako, L. 1991. Phyllopsora (Bacidiaceae). Flora Neotropica. Monogr. 55: 1-66.

Brodo, I. M.; SharnofF, S. D. \& Sharnof, S. 2001. Lichens of North America. Yale University Press.

Büdel, B. \& Scheidegger, C. 1996. Thallus morphology and anatomy. En T. Nash (Ed), Lichen Biology: 37-64. Cambridge University Press.

Coppins, B. J. \& Wolseley, P. 2002. Lichens of tropical forests. En R. Watling, J. C. Frankland, A. M. Ainsworth, S. Issac \& C. H. Robinson (Eds.), Tropical Mycology, 2. Micromycetes. CAB International.

Cornelissen, J. H. C. \& ter Steege, H. 1989. Distribution and ecology of epiphytic bryophytes and lichens in dry evergreen forest of Guyana. J. Trop. Ecol. 5: 131-150.

Eversman, Sh.; Johnson, C. \& Gustafson, D. 1987. Vertical distribution of epiphytic lichens on three tree species in Yellowstone National Park. Bryologist 90(3): 212-216.

Fanning, E.; Ely, J. S.; Lumbsch, H. T. \& Keller, H. W. 2007. Vertical distribution of lichen growth forms in tree canopies of Great Smoky Mountains National Park. Southeastern Naturalist 6(2): 83-88.
Frisch, A.; KalB, K. \& GrubE, M. 2006. Contributions towards a new systematics of the lichen family Thelotremataceae. Biblioth. Lichenol. 92: 1-556.

GallowaY, D. J. 1994. Studies on the lichen genus Sticta (Schreber) Ach.: I. Southern South American species. Lichenologist 26(3): 223-282.

Gradstein, R.; NADKARni, N.; KrÖMer, T.; Holz, I. \& NÖSKe, N. 2003. A protocol for rapid and representative sampling of vascular and non-vascular epiphyte diversity of tropical rain forest. Selbyana 24(1): 105-111.

Hale, E. Jr. 1976. A monograph of the lichen genus Pseudoparmelia Lynge (Parmeliaceae). Smithsonian Contributions to Botany 31: 1-62.

Hale, E. Jr. 1987. A monograph of the lichen genus Parmelia Acharius sensu stricto (Ascomycotina: Parmeliaceae). Smithsonian Contributions to Botany 66: 1-55.

Hinds, H. 1970. Vertical Distribution of Lichens on Aspens in Michigan. Bryologist 73 (3): 626-628.

HubBell, S. 2001. The Unified Neutral Theory of Biodiversity and Biogeography. Princeton University Press.

JAHns, H. M. 1988. The lichen thallus. En M. Galum (Ed.), Handbook of lichenology, 1: 95-143. Boca Raton: CRC Press.

JoHANSSON, D. 1974. Ecology of vascular epiphytic in West African rain forest. Acta Phytogeogr. Suec. 59: 1-129.

KIRK, P. M. 2008. Species Fungorum. http://www.speciesfungorum.org

Komposch, H. \& Hafellner, J. 2000. Diversity and vertical distribution of lichen in Venezuela tropical lowland rain forest. Selbyana 21(1, 2): 11-24.

Komposch, H. \& HafelLner, J. 2002. Life form diversity of lichenized fungi in an Amazon lowland rainforest. Biblioth. Lichenol. 82: 311-326. 
Lakatos, M.; Rascher, U. \& Büdel, B. 2006. Functional characteristics of corticolous lichens in the understory of a tropical lowland. New Phytologist 172: 679-695.

LANGE, O. L. 1969. Experimentell-ökologische Untersuchungen an Flechten der Negev-Wüste. I. CO2-Gaswechsel von Ramalina maciformis (Del.) Bory unter kontrollierten bedingungen im Laboratorium. Flora (Jena) 158: 324-359.

LÜCKING, R. \& Rivas-Plata, E. 2008. Clave y guía ilustrada para géneros de Graphidaceae. Glalia 1: 1-41.

Lumbsch, T.; NAsh, T. H. \& Messuti, M. I. 1999. A revision of Pertusaria species with hyaline ascospores in Southwestern North America (Pertusariales, Ascomycotina). Bryologist 102(2): 215-239.

MacArthur R. H \& Wilson, E. O. 1967. The theory of island biogeography. Monogr. Pop. Biol. Princeton Univ. Press.

McCune, B. \& Mefford, M. J. 1999. PC-ORD. Multivariate Analysis of Ecological Data. Version 5.0, MjM Software, Gleneden. Beach, Oregon, U.S.A.

Moberg, R. 1990. The lichen genus Physcia in Central and South America. Nord. J. Bot. 10: 319-342.

Montenegro, U. 1991. Clima. Atlas de Santiago de Cuba. BIOECO. MEGACEM.

MontFoort, D. \& EK, R. C. 1990. Vertical distribution and ecology of epiphytic bryophytes and lichens in lowland rain forest in French Guiana. Masters Thesis, Utrecht University, The Netherlands.

Nash, T. 1996. Lichen Biology. Cambridge University Press.

Purvis, W. 2000. Lichens. The Natural History Museum, London.

Reyes, O. J.; Pelicié, O.; C. Vinent; Labrada, L. M.; SemaNAT, E. \& Fornaris, E. 2005. Estudio fisionómico y funcional de la pluvisilvas montanas de la Gran Piedra, Cuba. Foresta Veracruzana 7(2): 7-14.
Rivas Plata, E.; LüCKInG, R. \& Lumbsch, H. T. 2007. When family matters: An analysis of Thelotremataceae (Lichenized Ascomycota: Ostropales) as bioindicators of ecological continuity in tropical forests. Biodiversity and Conservation 17: 1319-1351.

Rivas Plata, E.; Lücking, R.; Aptroot, A.; Sipman, H.; ChÁvez, J. L.; Umaña, L. \& Lizano, D. 2006. The genus Coenogonium (Ostropales: Coenogoniaceae), with a world-wide key and checklist and a phenotype-based cladistic analysis. A first assessment of the lichen biodiversity inventory in Costa Rica: Fungal Diversity 23: 255-321

Rosabal, D. \& Aragón, G. 2010. Líquenes epífitos en el matorral costero de la Reserva Ecológica Siboney-Juticí (Cuba). Bot. Complut. 34: 21-25.

Rosabal, D.; Burgaz, A. R. \& De la Masa, R. 2010. Diversity and distribution of epiphytic macrolichens on tree trunks in two slopes of the montane rainforest of Gran Piedra, Santiago de Cuba. Bryologist 113 (2):313-321.

Siegel, S. \& N. J. CASTELlÁN. 1995. Estadística no paramétrica: aplicada a las ciencias de la conducta. Editorial Trillas, México D. F. México.

Sipman, H. J. M. \& Harris, R.C. 1989. Lichens. En H. Lieth \& M. J. A. Werger (Eds.), Ecosystems of the world. 14B. Tropical rainforest ecosystem. Biogeographical and ecological studies, 15: 303-309. Amsterdan.

Wirth, M. \& M. E. HaLe, Jr. 1978. Morden-Smithsonian expedition to Dominica: The lichens (Graphidaceae). Smithsonian Contributions to Botany 40: 1-64.

WoLF, J. 1995. Non-vascular epiphyte diversity patterns in the canopy of an upper montane rain forest (2550-3670 m), central cordillera, Colombia. Selbyana 16:185-195. 